\title{
EXPERIMENTAL USE OF SATELLITE PICTURES IN NUMERICAL PREDICTION
}

\author{
E. PAUL MCCLAIN, MARY ANN RUZECKI, AND HAROLD J. BRODRICK
}

Meteorological Satellite Laboratory, U.S. Weather Bureau, Washington, D.C.

\begin{abstract}
Errors in operational forecasts produced by high-speed electronic computers can be classed broadly into two categories: (1) those resulting from inadequacies of the dynamic model, and (2) those resulting from poor specification of the initial fields. Many regions of the Northern Hemisphere, particularly oceanic areas, are poorly observed in terms of conventional meteorological data, especially upper-air data. The SINAP (Satellite Input to Numerical Analysis and Prediction) Project at the Weather Bureau's Meteorological Satellite Laboratory has been working to develop techniques for incorporating information derived from satellite cloud pictur es into the operational numerical analysis in data-sparse areas.

Trial reanalyses of the National Meteorological Center (NMC) 500-mb. stream function analysis, or its Laplacian, were performed for data-sparse areas of the central and eastern Pacific Ocean using an analysis modification technique consisting of two steps: (1) inferring features of the flow pattern or of the field of large-scale vertical motion from an interpretation of the TIROS-viewed cloud patterns, and (2) modifying the 500-mb. analyses to produce an appropriate vorticity advection field. Underlying this method are certain simplifying assumptions about the relation of the cloud field to the vertical motion field on the one hand, and of the vertical motion to the vorticity advection on the other.

Application of the method and the results obtained are illustrated for one case. Thirty-six-hr. barotropic forecasts were run from both the original NMC analysis and the SINAP modified analysis and then compared with the verifying chart. Verification statisties, such as the root mean square (RMS) error of the stream values and of the vector geostrophic wind, are presented for the case jllustrated and for five additional cases. Significant reductions in forecast error were achieved in most cases, the overall average reduction in the RMS error of the wind being 5.4 percent.
\end{abstract}

\section{INTRODUCTION}

The TIROS satellite system has provided meteorologists with one of the most intriguing tools for cloud observations yet invented. Since April 1, 1960, over 360,000 usable pictures have been taken by TIROS cameras, and much information has been gleaned from careful studies of cloud formations and placements in association with analyses of other types of meteorological data. The meteorological literature has been enriched by contributions of investigators who have employed the photographs to examine cloud forms and patterns in considerable detail. In spite of these studies relatively little operational use has yet been made of satellite weather data, although this may be due in part to the circumstance that the TIROS satellites are not operational but research and developmental in nature, and that the geographic coverage provided by them has been restricted and somewhat irregular. In particular there has been little research into the objective use of satellite photographs as input data for numerical experiments using high-speed electronic computers.

A paper by Bristor and Ruzecki [1] outlines a method of using the photographs as numerical input for atmospheric flow prediction models. Miller [8] presents another method of using TIROS photographs subjectively to deter- mine the $500-\mathrm{mb}$. flow patterns in mid-latitudes and then introduces the flow directions into a numerical analysis computer program. But it is clear that much work remains in the area of deriving objective, quantitative information from satellite cloud photographs. For this purpose, Project SINAP (Satellite Input to Numerical Analysis and Prediction) was initiated at the Meteorological Satellite Laboratory (MSL) in October 1963. The basic goal of Project SINAP is to develop the use of satellite data in data-sparse regions. Improvements in numerical forecasting can be achieved in at least two ways: improved numerical models can be developed, or a more accurate analysis of the initial state of the atmosphere can be made. Project SINAP has concerned itself with the latter possibility. Following the general method developed by Ruzecki [10], a series of 500-mb. stream function fields were reanalyzed on the basis of satellite pictures in four synoptic situations. To test the usefulness of these reanalyses $36-\mathrm{hr}$. forecasts were run on both the SINAP and the National Meteorological Center (NMC) numerical analyses and compared.

The purpose of this paper is to describe the reanalysis technique and to discuss in some detail one of the case studies completed in Project SINAP. Statistical verifications for this and five additional cases are included in the discussion. 


\section{BASIS FOR REANALYSIS}

There are two basic steps in the reanalysis procedure developed by the SINAP group: (1) infer information about the 500-mb. flow pattern, or about the large-scale, mid-tropospheric vertical motion, from cloud patterns photographed by the satellite; (2) translate this information into terms that enable one to modify the initial 500$\mathrm{mb}$. analysis where there appears to be an inconsistency between the flow pattern and the cloud pattern. The first of these steps draws upon our limited knowledge relating satellite-viewed cloud patterns to the wind and weather systems analyzed on surface and upper-air charts. Widger [13] has synthesized the results of a number of studies relating to the extratropical vortex patterns seen by TIROS. When his models are combined with longestablished synoptic models of surface frontal cyclones and their associated upper-air systems, it is often possible to diagnose the general configuration of the $500-\mathrm{mb}$. flow or to infer the nature of the large-scale vertical motion field. The results of other studies of the meteorological interpretation of synoptic-scale cloud patterns seen in satellite pictures $[2,4,7,9]$ or of the inference of wind information from the cloud appearance [6] have also proven useful in accomplishing the first stage of the SINAP reanalysis.

Step two in the SINAP reanalysis procedure entails modification of the $500-\mathrm{mb}$. field(s). Some of the adjustments are relatively simple and direct, e.g., the introduction or repositioning of a circulation center, or the altering of the direction of the flow in a region, or the relocation of the axis of maximum wind. The connection between the satellite-inferred areas of large-scale vertical motion and the 500-mb. flow is more complicated, however, and requires some elaboration.

It is well known that areas of upward (downward) motion in the middle and upper troposphere are associated with areas of strong positive (negative) vorticity advection at these levels. The "omega equation" (see Appendix) relates the vertical motion field to the vertical variation of vorticity advection and the Laplacian of the thickness advection. The usual relationship between a sea level cyclone and its associated $500-\mathrm{mb}$. trough is such that both vorticity advection and thickness advection make their maximum contribution to the vertical motion in nearly the same sectors of the cyclone [12]. Cyclonic vorticity advection increases upward through the middle and upper troposphere in the pre-trough region to produce ascent over and northeast of the surface cyclone center. The Laplacian of the thickness advection also contributes to ascent in the eastern or northeastern sectors of the cyclone where warm advection attains its maximum. Similarly, anticyclonic vorticity advection in the post-trough region and the maximum cold advection found in the western or southwestern sector of the cyclone contribute to descent in these sectors. The general influence, therefore, of the thickness advection term, over and above that of the vorticity advection term, is to increase the magnitude of both the ascent and the descent, and to spread the ascent north- eastward and the descent southeastward. For simplicity's sake, therefore, and also because a barotropic forecast model was used in these experiments, all the ascent inferred from the cloud patterns was attributed to the effects of vorticity advection only.

The flow patterns at $500 \mathrm{mb}$. may be given in terms of a stream function, $\psi$, defined such that the (non-divergent) wind is given by

$$
u=-\partial \psi / \partial y, \quad v=\partial \psi / \partial x
$$

The stream function field may be derived from the topography of the isobaric surface by means of the socalled "balance-equation" [3]:

$$
f \nabla^{2} \psi+\nabla \psi \cdot \nabla f-2\left[\left(\partial^{2} \psi / \partial x \partial y\right)^{2}-\left(\partial^{2} \psi / \partial x^{2}\right)\left(\partial^{2} \psi / \partial y^{2}\right)\right]=g \nabla^{2} z
$$

where $f=2 \Omega \sin \phi$, the Coriolis parameter.

The balance equation provides a means of determining the wind from the height field $z$ without employing the geostrophic assumption, but its solution requires the use of relaxation methods. Computer programs written by the MSL and by the NMC are used to obtain from the history data tapes print-outs of not only the stream function $\hat{\psi}$ field, but also of the finite-difference Laplacian $\nabla^{2} \hat{\psi}$ and Jacobian $J\left[\hat{\psi}, \nabla^{2} \hat{\psi}\right]$ fields, where $\psi \equiv\left(g / f_{0}\right) \hat{\psi}, \nabla^{2} \equiv$ $(m / d)^{2} \nabla^{2}$, and $J \equiv(m / 2 d)^{2} \mathrm{~J}$. The relative vorticity and vorticity advection are then written, respectively, as follows:

and

$$
\zeta=\nabla^{2} \psi=\frac{m^{2} g}{d^{2} f_{0}} \nabla^{2} \hat{\psi}
$$

$$
-\mathbf{V} \cdot \nabla \zeta=J\left(\psi, \nabla^{2} \psi\right)=\frac{m^{4} g^{2}}{4 d^{4} f_{0}^{2}} J\left(\hat{\psi}, \nabla^{2} \hat{\psi}\right)
$$

where $f_{0}$ is $f$ at $\phi=45^{\circ} \mathrm{N}$., $d$ is the NWP grid distance of $381 \mathrm{~km}$. at $\phi=60^{\circ} \mathrm{N}$, and $m=\left(1+\sin 60^{\circ}\right)(1+\sin \phi)^{-1}$, the map factor.

\section{CASE SELECTION AND REANALYSIS PROCEDURE}

Since our objective is the improvement of $500-\mathrm{mb}$. initial analyses in data-sparse areas, the following procedure of case selection has been used. The error charts (forecast minus verifying) are examined for forecast errors of more than $150 \mathrm{~m}$. located in the eastern Pacific area. The system associated with the error is located; and if $36 \mathrm{hr}$. earlier it was situated in a data-sparse region, the TIROS coverage maps are examined. If the satellite pictures cover the error source region, then the synoptic situation is examined further to determine the need for reanalysis in the light of the satellite data. When all criteria are met a case study is undertaken. As discrepancies between the NMC flow pattern analyzed in a sparse data area and a cloud system viewed by the satellite are noted during a current-weather map discussion, possible reanalyses are then discussed, or actually performed, on an essentially "real-time" basis."

\footnotetext{
I Such was the circumstance in the A pril 1964 case presented in the next section. The initial stream reanalysis was based on an operational nephanalysis and a relatively poor photo-facsimile print of a single frame. The computational part, including the forecast, photo-facsimile print of a single frame. The computational part, including the forecast,
was accomplished some days later. A second reanalysis was performed some months Iater when high-quality prints from archival film were available.
} 
Six trial reanalyses, based upon four synoptic situations, were completed in Project SINAP during the first year. In one case more than one geographical area was reanalyzed and the results were compiled separately. In another case two separate reanalyses were made of the same situation by two meteorologists, and these results are shown as. separate studies. In all instances the area of reanalysis was located over data-sparse regions of the Pacific Ocean.

The data source was the NMC archival history data tapes. These tapes contain the following information: the first guess field, raw teletypewriter data that have been sorted and checked by a computer program, height and temperature analyses for levels from $1000 \mathrm{mb}$. to $100 \mathrm{mb}$., and $500-\mathrm{mb}$. forecasts out to $48 \mathrm{hr}$. The vertical velocity field at $650 \mathrm{mb}$. and the $500-\mathrm{mb}$. stream function and isotach analyses are also available from the tape. Any of these fields, and derivative fields such as finite difference Laplacians and Jacobians, can be printed-out and used as diagnostic tools with the TIROS cloud photographs and nephanalyses.

When a synoptic situation is reanalyzed either the stream function field or its derivative, the Laplacian field, is altered. The area altered takes the form of a rectangular subset of the NWP grid consisting of 100 or more grid points. If the stream field is modified, the altered part is melded into the original NMC analysis in the surrounding area. Laplacian and Jacobian fields are then derived and examined to see if the desired vorticity and vorticity advection patterns have resulted from the modification. If some further adjustments are deemed necessary, the process can be repeated; or alternatively the Laplacians within the reanalysis area can be modified. Once the altered part of the Laplacian field has been melded into the initial NMC Laplacian field neighboring it, relaxation yields the new SINAP stream field, and a SINAP Jacobian field can then be derived. Certain restraints are necessary in the application of these reanalysis procedures. One must take care not to violate any existing upper-air data within or near the borders of the reanalysis area. In using the alternate approach of modifying a portion of the Laplacian field, it is necessary to keep unchanged the average (algebraic) value of the Laplacian within the reanalysis area. Should there be a net gain (loss) of vorticity within this region, relaxation will result in a stream field in which the stream values have been lowered (raised) over the entire hemispheric grid. Experience has indicated that most imbalances resulting from the initial reanalysis can be removed by very minor adjustments (one unit or two) at numerous grid points spread over the entire reanalysis area or near the boundaries.

When the final reanalysis has been arrived at, a $36-\mathrm{hr}$. numerical forecast is run. The forecast model used in SINAP is the one-level barotropic model developed by NMC. This model was chosen because of its simplicity$500 \mathrm{mb}$. is the only input level-and because input is readily available with no requirement for data preprocess- ing or change of format. A 36-hr. forecast is run from the original NMC numerical analysis also.

The various ways in which the NMC and SINAP forecasts are compared with the verifying analysis and with each other in order to evaluate the consequences of the SINAP modifications are presented and discussed in the next two sections in connection with an actual trial reanalysis.

\section{CASE OF APRIL 7, 1964}

On April 6, 1964, at about 1835 GMT the cameras of TIROS VII photographed a spiral-like cloud system near $38^{\circ} \mathrm{N}$., $151^{\circ} \mathrm{W}$. (see mosaic of fig. 1 and the nephanalysis of fig. 2). The nearest rawinsonde stations are in the Hawaiian Islands and the weather ships at $30^{\circ} \mathrm{N}$., $140^{\circ} \mathrm{W}$. and $50^{\circ} \mathrm{N}$., $145^{\circ} \mathrm{W}$. (see fig. 3). A weak, open wave was analyzed near $39^{\circ} \mathrm{N}$., $148^{\circ} \mathrm{W}$. on the $0000 \mathrm{GMT}$, sea level chart (not reproduced), and only the weakest indication of a short-wave trough was analyzed in this vicinity on the $500-\mathrm{mb}$. chart (fig. 3). The left half of figure 4 gives the Laplacian field derived from the NMC stream field, and it is evident that there is no cyclonic vorticity analyzed in the vicinity of the spiral. The NMC Jacobian field (fig. 5) likewise shows no cyclonic vorticity advection associated with this storm system.

The first trial reanalysis, noted in the previous section as having been done on a real-time basis, consisted of altering the stream function values at grid points in the neighborhood of the spiral. The flow pattern was changed so as to produce a cyclonic vorticity maximum there, and to produce a positive vorticity advection maximum in the region northeast of the vortex where the thick, multi-layer stratiform cloud was interpreted to exist (see figs. 1 and 2). A second reanalysis, at a much later time, was performed by altering the Laplacian field. Although there were minor differences in the flow patterns of the two reanalyses, the resulting $36-\mathrm{hr}$. barotropic forecasts were nearly identical, and only the charts for the second trial are given here. The right side of figure 4 gives the SINAP Laplacian field, and the right side of figure 5 gives the SINAP Jacobian field. Note that a cyclonic vorticity maximum has been introduced near and just south of the spiral center (the position of which has been adjusted to allow for the time difference between the TIROS pictures and map time). This in turn has introduced an area of cyclonic vorticity advection to the northeast of the spiral where the strongest vertical motion was inferred from the appearance of the clouds. Relaxation of the adjusted Laplacian field yielded the SINAP stream field shown by the dashed lines in figure 3 .

The most difficult step is probably that of deciding just how much to change the vorticity, and thereby the vorticity advection, in a given case. This particular cloud spiral has the appearance of one associated with a young occlusion [4], and the area of thick, or multi-layer, 


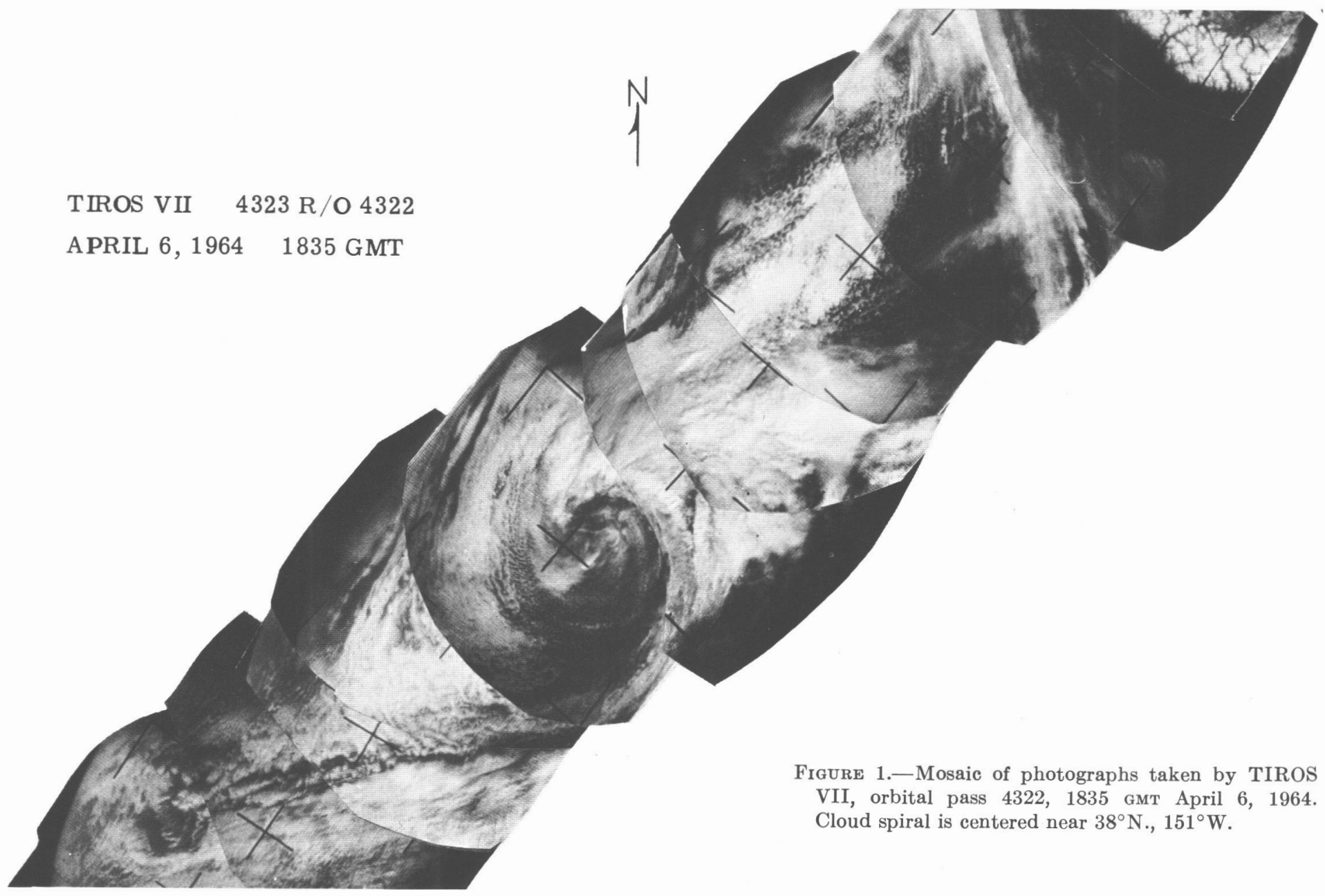

stratiform cloud is fairly small. An elongated area of relatively weak positive vorticity advection was depicted in the region of the weak-appearing frontal cloud zone extending southwestward from the eastern periphery of the spiral. Negative vorticity advection appears, however, in the area of stratocumuliform cloud southwest and west of the spiral center.

The question of whether or not modifications introduced on the basis of satellite information significantly "improve" the analysis is a formidable one. One recourse is to run numerical forecasts from both the NMC and SINAP initial stream fields and compare the results with the verifying $\mathrm{NMC}$ analysis. It would be preferable to have the verifications centered in a dense-data area, but this frequently is not possible, so there may be some question as to the accuracy of the verifying analysis. Excluding such influences as diabatic effects, which are not included in either prediction model, errors in baroclinic forecasts are due largely to poor specification of the initial state, whereas errors in barotropic forecasts may be due to either this or baroclinic effects, or both. In the SINAP experiments error charts derived from NMC barotropic and baroclinic forecasts were compared, and only.those cases in which the baroclinic forecast errors were also large were included in the trials. It would seem to follow, in any case, that better specification of the initial state should lead to better baroclinic forecasts as well as to better barotropic ones.

In the April 1964 case it is evident that the NMC forecast (fig. 6) fails to show the trough system to the southeast of the major low center in the Aleutians, whereas this feature is depicted in the SINAP forecast (fig. 7), albeit not perfectly. Further comparison of the two forecasts is afforded by the error fields shown in figures 6 and 7 , where it is seen that the maximum error in the SINAP forecast is about half that in the NMC forecast. Another type of chart, designated the Forecast Improvement Chart (F.I. Chart), is readily obtained by having the following computed and printed-out at each grid point:

F.I.=|NMC FCST.-VERIF.|-|SINAP FCST.-VERIF. Positive F.I. values signify improvement of the forecast by the SINAP reanalysis, whereas negative ones mean impairment. Figure 8 presents the F.I. Chart for the April 1964 case. The improvement maximum of about $190 \mathrm{~m}$. is directly associated with the vorticity maximum introduced in the analysis southwest of there $36 \mathrm{hr}$. earlier. Other lesser areas, some of improvement and some of impairment, are not so easily accounted for, but most result apparently from changes in the analysis that 


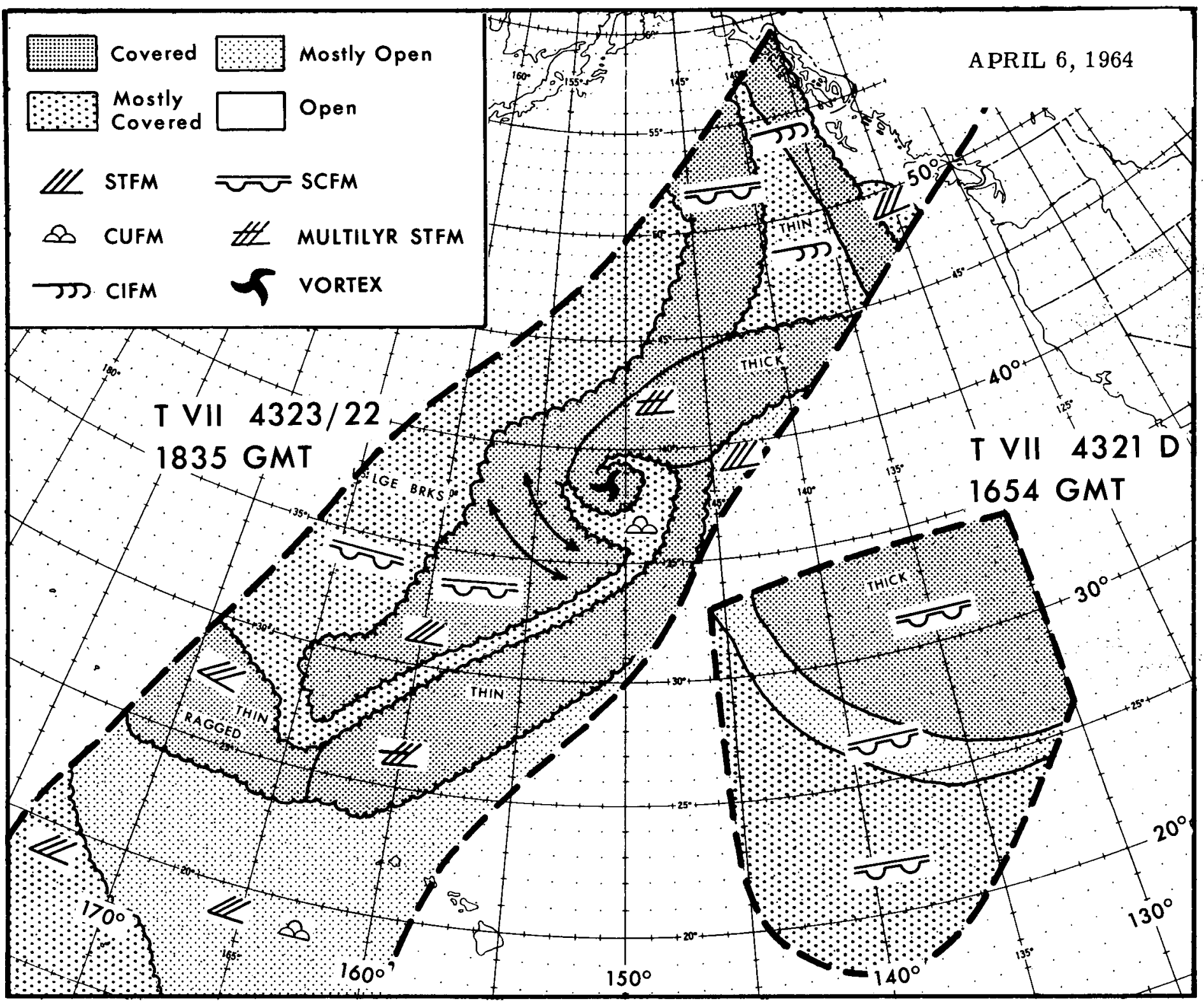

Figure 2.- Nephanalysis constructed for portions of the areas viewed by TIROS VII, passes 4321 (1654 GMT) and 4322 (1835 GMT) on April 6, 1964. This type of nephanalysis puts the major emphasis on those aspects of the cloud patterns most useful in SINAP reanalyses.

occur near the boundary of the reanalysis area where the new analysis is melded with the old.

\section{VERIFICATION STATISTICS}

In addition to the verification charts described above, a number of statistics were computed also. Root mean square (RMS) errors and correlation coefficients were calculated from the NMC and SINAP barotrophic forecasts for the following fields: stream function, vector geostrophic wind, stream Laplacian, and stream Jacobian. Table 1 presents a comparison of the RMS errors of the

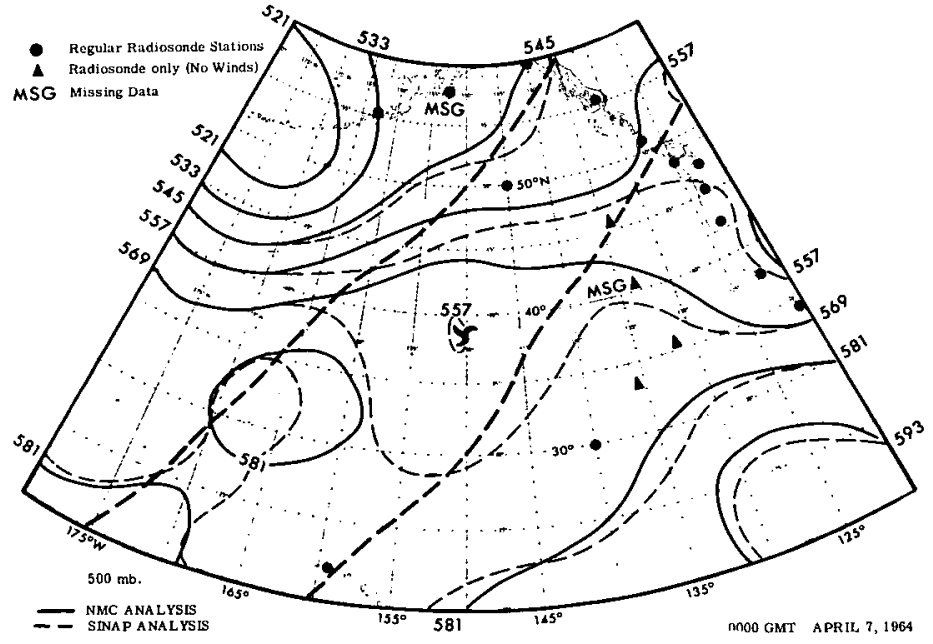

FIgURE 3.-Comparison of NMC numerical analysis (solid lines) and the SINAP reanalysis (dashed lines) at the 500-mb. level, 0000 GMT April 7, 1964. Stream function $\hat{\psi}$ isopleths are labeled in tens of meters. Heavy broken lines enclose that portion of the area viewed by TIROS VII on pass 4322 


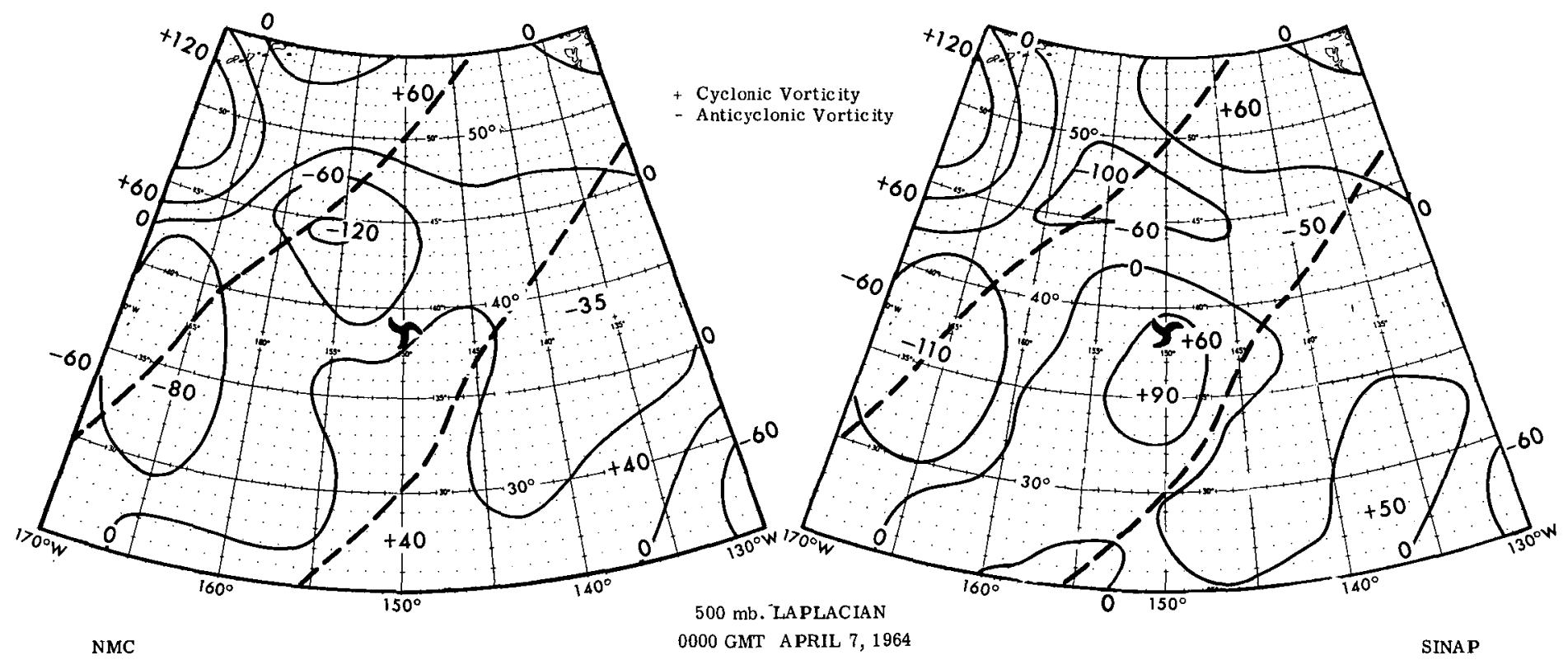

FIGURE 4.-Comparison of NMC and SINAP finite-difference Laplacian $\nabla^{2} \hat{\psi}$ fields corresponding to the stream function fields shown in figure 3. The isopleths are labeled such that positive values correspond to cyclonic vorticity and negative to anticyclonic, but they do not contain the factor $m^{2} a / d^{2} f_{n}$ and thus are in units of meters.

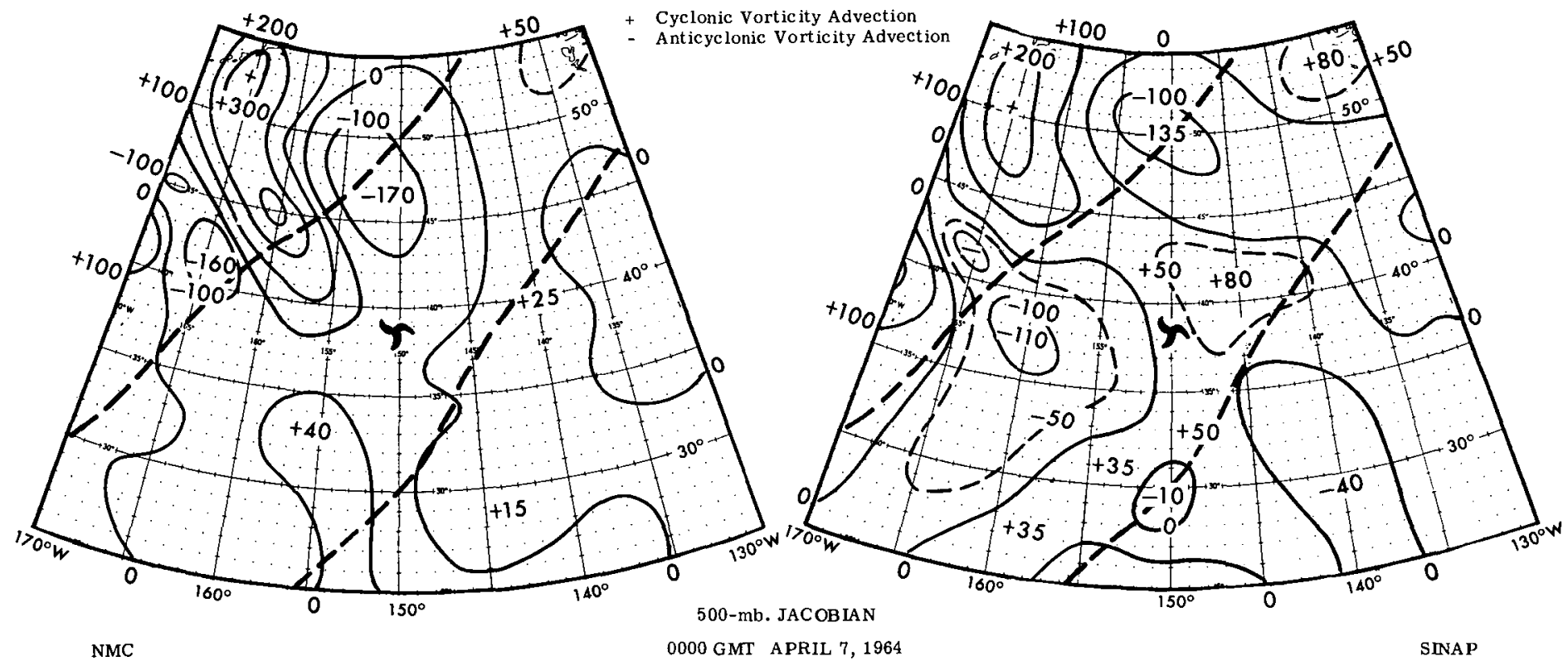

FIGURE 5.-Comparison of NMC and SINAP finite-difference. Jacobian $\rfloor\left[\hat{\psi}, \nabla^{2} \hat{\psi}\right]$ fields corresponding to the stream function fields shown in figure 3. The isopleths are labeled such that positive values correspond to cyclonic vorticity advection and negative to anticyclonic, but they do not contain the factor $m^{4} g^{2} / 4 d^{4} f_{0}^{2}$ and thus are in units of $10^{2} \mathrm{~m}^{2}$.

TABLE 1.-Root mean square (RMS) error comparisons

\begin{tabular}{|c|c|c|c|c|c|c|}
\hline \multirow{2}{*}{ Case } & \multicolumn{3}{|c|}{ Stream function (m.) } & \multicolumn{3}{|c|}{$\underset{(\mathrm{kt} .)}{\text { Vector geostrophic wind }}$} \\
\hline & NMC & SINAF & $\begin{array}{c}\text { Percent } \\
\text { improve- } \\
\text { ment }\end{array}$ & NMC & SINAP & $\begin{array}{c}\text { Percent } \\
\text { improve- } \\
\text { ment }\end{array}$ \\
\hline $\begin{array}{l}\text { Nov. 1, } 1963 \text { (E. Pac.) } \\
\text { Nov. 1, } 1963 \text { (W. Pa.)... } \\
\text { Nov. 5, } 1963 \\
\text { Jan. } 15,1964 \\
\text { Apr. .7, 1964 (Trials i and 2 } \\
\text { averaged) }\end{array}$ & $\begin{array}{l}55.3 \\
55.3 \\
65.2 \\
83.2 \\
93.0\end{array}$ & $\begin{array}{l}53.6 \\
48.7 \\
59.9 \\
72.6 \\
72.3\end{array}$ & $\begin{array}{r}+3.1 \\
+11.9 \\
+8.1 \\
+12.8 \\
+22.3\end{array}$ & $\begin{array}{r}9.4 \\
9.4 \\
9.9 \\
11.4 \\
14.2\end{array}$ & $\begin{array}{r}9.3 \\
9.2 \\
9.7 \\
10.8 \\
12.0\end{array}$ & $\begin{array}{r}+1.1 \\
+2.1 \\
+2.0 \\
+5.3 \\
+15.5\end{array}$ \\
\hline A verage & 70.4 & 61.4 & +11.6 & 10.9 & 10.2 & +5.4 \\
\hline
\end{tabular}

first two of these fields for the six cases individually ${ }^{2}$ and for the average of all cases. For simplicity a rectangular array consisting of 546 grid points, an area somewhat larger than that affected by the analysis changes, was used as the verification subset in all cases.

In view of the crudity of the present technique, these results are most encouraging. To provide at least some kind of basis for assessing the possible significance of these

2 Since, as was mentioned in section 4, the results of Trials 1 and 2 of the $A$ pril 7,1964 , case were nearly identical, they were averaged before inclusion in table 1. 


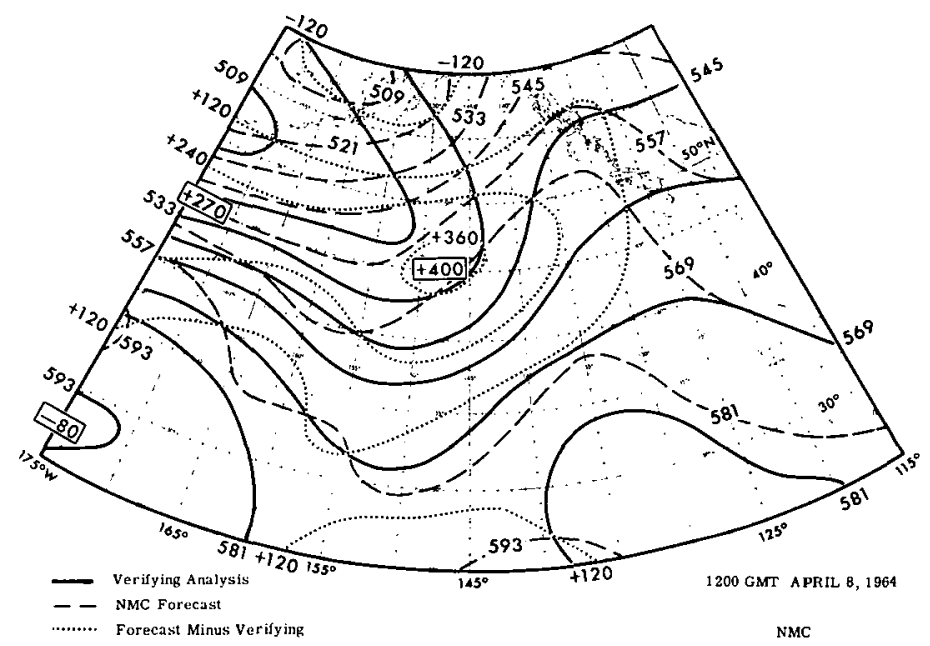

FIgURe 6.-Comparison of the verifying stream function $\hat{\psi}$ analysis (1200 GMT April 8, 1964) with the 36-hr. barotropic forecast based on the NMC analysis. Isopleths are labeled in tens of meters. Also shown is the corresponding error field (NMC forecast minus verifying) in meters. Values in boxes show location and magnitude of maximum errors.

results, we might compare them with some RMS error reductions achieved when the NMC 3-level model was tested against the operational barotropic model. Cressman [5] presents a comparison of $36-\mathrm{hr}$., $500-\mathrm{mb}$. barotropic and 3-level model forecasts made during the period April 13-June 16, 1962. In Area I, which covers principally North America, the reduction in wind error was from 14 to $13 \mathrm{kt}$. (7.1 percent), and in height error from 165 to $149 \mathrm{ft}$. (9.7 percent). The forecast error reductions achieved in the SINAP experiments, which ranged from negligible in some of the cases to the rather large values shown for the April 1964 case, are not strictly comparable to those just cited, of course, since the SINAP cases are mostly a small developmental sample. Only one case (April 7, 1964, Trial 1) was really an operational evaluation of the method. Future SINAP experiments, therefore, should be designed such that the analyst has no knowledge of either the verifying analysis or the accuracy of the NMC forecast.

\section{SUMMARY AND CONCLUDING REMARKS}

During the first year of Project SINAP six case studies were completed and documented, all reanalyses being performed in regions of sparse data, namely the Pacific Ocean. These first attempts to improve the initial operational analysis by the use of satellite information have shown positive results. Percentage improvements in the RMS error of the vector geostrophic wind varied from 1 percent to 15.5 percent, averaging 5.4 percent.

Statistics alone, however, do not reflect the total progress made during SINAP's first year. The catalog of descriptions and modifications based upon TIROS cloud photographs has been expanded, and the experience gained by associating TIROS-viewed cloud patterns with the

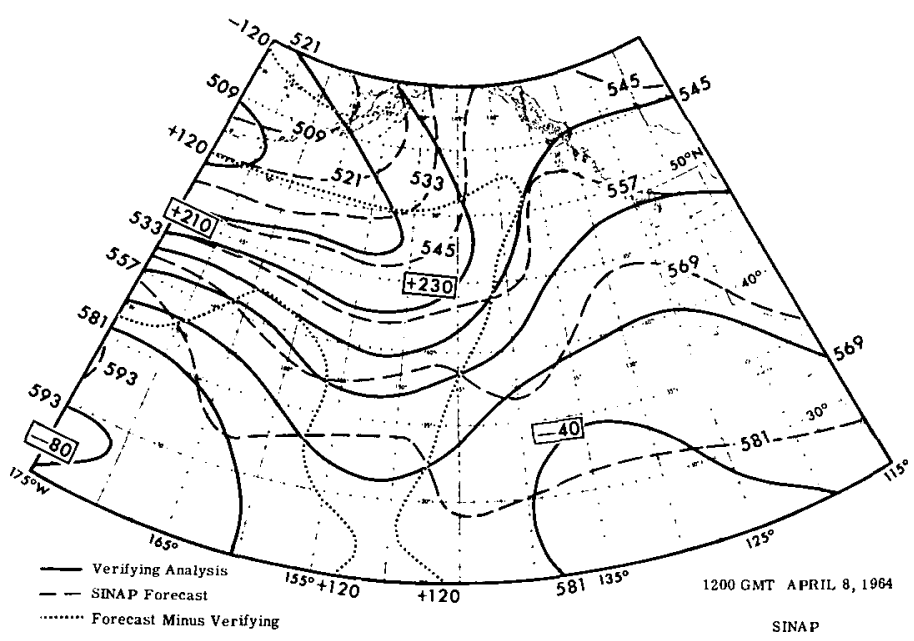

FIGURe 7.-Comparison of the verifying stream function $\hat{\psi}$ analysis (1200 GMT April 8, 1964) with the 36-hr. barotropic forecast based on the SINAP reanalysis. Also shown is the error field (SINAP forecast minus verifying). Values in boxes show location and magnitude of maximum errors.

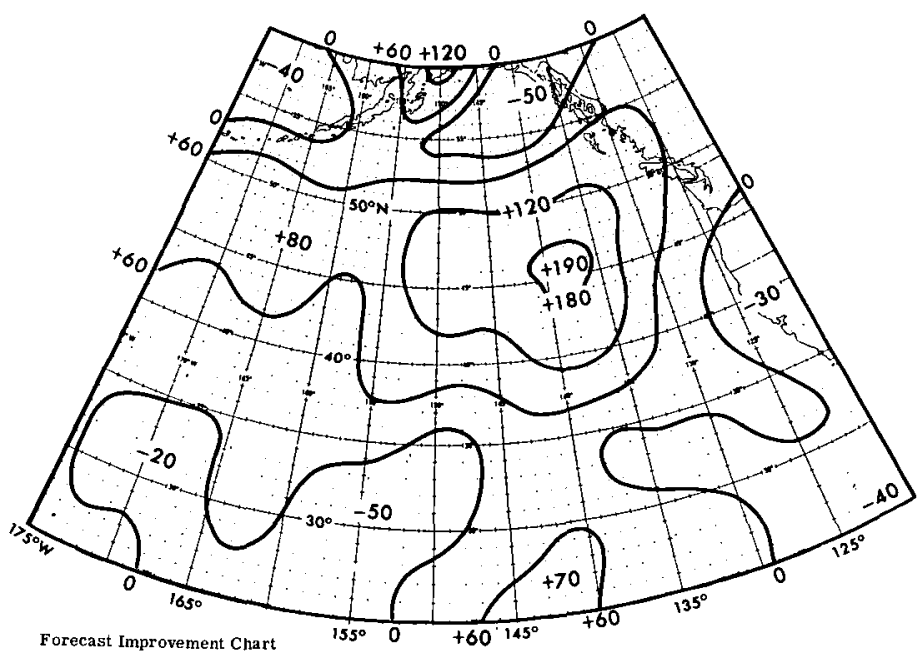

$1200 \mathrm{GMT}$ APRIL 8,1964

Figure 8.-Forecast Improvement Chart for forecasts verifying at 1200 GMT April 8, 1964. This chart shows the difference in the absolute error fields (NMC minus SINAP) shown in figure 6. Isopleths are labeled in meters.

corresponding flow patterns at $500 \mathrm{mb}$. has given the analysts increased confidence in the appropriateness of the modifications they make in the analyses.

The number of SINAP cases completed to date is small, and this is due in large part to the greatly restricted coverage afforded by the initial TIROS series. This limitation should be removed with the substantially expanded coverage designed for the new series beginning with Nimbus, TIROS IX and the Weather Bureau's TIROS Operational Satellite (TOS) series to follow.

Future plans for SINAP include the development of more systematic and increasingly objective rules for accomplishing the step from picture to reanalysis. Ex- 
periments are under way to develop ways of making explicit use of the Laplacian of thickness advection contribution to vertical motion, since this will also be useful in any modification of SINAP procedures for baroclinic forecast models. The inclusion of data from the TIROS and Nimbus radiation experiments in SINAP reanalyses will also be attempted. Rectified multi-pass, digitized cloud mosaics to be computer produced with the advent of TOS operations offer challenging possibilities for developing various types of satellite data inputs to numerical analysis routines.

\section{APPENDIX}

The following summarizes a recent and unusually clear interpretation of the so-called "omega-equation" by Sanders [11]:

$$
\left(\nabla^{2}+\frac{f_{0}^{2}}{\sigma} \frac{\partial^{2}}{\partial p^{2}}\right) \omega=\frac{f_{0}}{\sigma} \frac{\partial}{\partial p}(\mathbf{V} \cdot \nabla \eta)-\frac{1}{\sigma} \nabla^{2}\left(\mathbf{V} \cdot \nabla \frac{\partial \Phi}{\partial p}\right)
$$

where pressure is used as the vertical coordinate. In this expression, $\Phi$ is the geopotential of an isobaric surface; $\eta$ is the absolute vorticity, $\zeta+f ; f_{0}$ is a standard value of the Coriolis parameter; $\sigma$ is a stability parameter $(\partial \ln \theta / \partial p)$ $(\partial \Phi / \partial p)$ that is assumed to vary only with pressure; $\theta$ is potential temperature; and $\omega$ is the vertical motion, $d p / d t$. This is a geostrophic formulation, and thus it is not applicable for the smaller synoptic scales of motion; furthermore, the effects of diabatic heating are not taken into acount.

With the application of suitable boundary conditions at the bottom and top of the atmosphere, the omega-equation may be given a fairly simple and direct interpretation in terms of quantities readily obtainable from conventional meteorological analyses or as by-products of numerical flow analysis and prediction models in operational use. The left side of this equation may be viewed as a sort of three-dimensional Laplacian operator that characterizes the configuration of the three-dimensional field of vertical motion. When the argument is restricted to the maximum point in an area of ascent (negative $\omega$ ), with the meteorologically realistic assumption that this point is surrounded by a family of more-or-less spherical equivalue $\omega$ surfaces (this ensures that both components of the operator have the same sign), then positive values of the operator tend to be found throughout the region in the vicinity of the maximum point. Analogous arguments could be presented for inferring negative values of the operator in the region immediately surrounding the maximum point in an area of descent (positive $\omega$ ). The essence of this equation is, assuming a fairly simple but realistic geometry for the vertical motion field, that the total vertical motion can be interpreted as the sum of two partial fields, one due to the rate of change of vorticity advection along the vertical and the other to the horizontal Laplacian of the thickness advection. In regions where cyclonic vorticity advection increases upward (or anticyclonic vorticity advection decreases upward), the first term on the right of the equation contributes to ascent. Where the thickness and flow patterns indicate a maximum of warm advection, the second term on the right contributes to ascending motion also. Conversely, in regions where anticyclonic vorticity advection increases upward and cold advection attains a maximum both terms contribute to descending motion.

\section{ACKNOWLEDGMENTS}

We wish to acknowledge the assistance of V. J. Oliver, R. K. Anderson, and T. I. Gray, who participated in many of the early discussions and trial reanalyses. Messrs. Oliver and Anderson also performed the Trial 1 reanalysis of the April 1964 case reported on in this article. The authors also wish to thank Dr. S. Fritz who has provided stimulating discussions and continuing encouragement. They are grateful to L. F. Hubert and C. L. Bristor for a critical reading of the manuscript.

\section{REFERENCES}

1. C. L. Bristor and M. A. Ruzecki, "TIROS I Photographs of the Midwest Storm of April 1, 1960," Monthly Weather Review, vol. 88, Nos. 9-12, Sept.-Dec. 1960, pp. 315-326.

2. H. J. Brodrick, "TIROS Cloud Pattern Morphology of Some Mid-Latitude Weather Systems," Meteorological Satellite Laboratory Report No. 24, U.S. Weather Bureau, 1964, 31 pp.

3. J. Charney, "The Use of the Primitive Equations of Motion in Numerical Forecasting," Tellus, vol. 7, No. 1, Feb. 1955, pp. 22-26.

4. J. H. Conover, "Cloud Interpretation from Satellite Altitudes," CR Research Note 81, Air Force Cambridge Research Laboratories, 1962, 77 pp.; and Supplement 1, 1963, 19 pp.

5. G. P. Cressman, "A Three-Level Model Suitable for Daily Numerical Forecasting," National Meteorological Center, Technical Memorandum No. 22, U.S. Weather Bureau, 1963, $43 \mathrm{pp}$.

6. C. O. Erickson, "Satellite Photographs of Convective Clouds and Their Relation to the Vertical Wind Shear," Monthly Weather Review, vol. 92, No. 6, June 1964, pp. 283-296.

7. A. F. Krueger and S. Fritz, "Cellular Cloud Patterns Revealed by TIROS I," Tellus, vol. 12, No. 1, Feb. 1961, pp. 1-7.

8. F. R. Miller, "An Application of TIROS Cloud Observations in Sparse Data Regions," Monthly Weather Review, vol. 91, No. 9, Sept. 1963, pp. 433-451.

9. V. J. Oliver, R. K. Anderson, and E. W. Ferguson, "Some Examples of the Detection of Jet Streams from TIROS Photographs," Monthly Weather Review, vol. 92, No. 10, Oct. 1964, pp. 441-448.

10. M. A. Ruzecki, "The Use of Satellite Cloud Photographs in Numerical Weather Prediction," Meteorological Satellite Laboratory Report No. 23, U.S. Weather Bureau, 1963, 22 pp.

11. F. Sanders, "Further Research Directed Toward the Study of Relations of Atmospheric Flow to Weather," Massachusetts Institute of Technology, Final Report, Contract No. AF19(604)-8373, 1963, Appendix C., 14 pp.

12. Staff, National Weather Analysis Center, Synoptic Meteorology as Practiced by the National Meteorological Center (The NAWAC Manual), Washington, D.C., October 1960 (Part I, 33 pp.) and Nov. 1961 (Part II, 38 pp.)

13. W. K. Widger, Jr., "A Synthesis of Interpretations of Extratropical Vortex Patterns as Seen by TIROS," Monthly Weather Review, vol. 92, No. 6, June 1964, pp. 263-282.

[Received March 17, 1965; revised April 28, 1965] 